\title{
On the inflaton potential from antibranes in warped throats
}

\author{
Iosif Bena, ${ }^{a}$ Gregory Giecold, ${ }^{a}$ Mariana Graña ${ }^{a}$ and Nick Halmagyi ${ }^{a, b}$ \\ anstitut de Physique Théorique, CEA Saclay, CNRS URA 2306, \\ F-91191 Gif-sur-Yvette, France \\ ${ }^{b}$ Laboratoire de Physique Théorique et Hautes Energies, Université Pierre et Marie Curie, \\ CNRS UMR 7589, \\ F-75252 Paris Cedex 05, France \\ E-mail: iosif.bena@cea.fr, gregory.giecold@cea.fr, \\ mariana.grana@cea.fr, halmagyi@lpthe.jussieu.fr
}

ABSTRACT: We compute the force between a stack of smeared antibranes at the bottom of a warped throat and a stack of smeared branes at some distance up the throat, both for anti-D3 branes and for anti-M2 branes. We perform this calculation in two ways: first, by treating the antibranes as probes in the background sourced by the branes and second, by treating the branes as probes in the candidate background sourced by the antibranes. These two very different calculations yield exactly the same expression for the force, for all values of the brane-antibrane separation. This indicates that the force between a brane and an antibrane is not screened in backgrounds where there is positive charge dissolved in flux, and gives a way to precisely compute the inflaton potential in certain string cosmology scenarios.

KEYWORDS: Gauge-gravity correspondence, dS vacua in string theory

ArXiv EPRINT: 1011.2626 


\section{Contents}

1 Introduction and motivation $\quad 1$

2 Computing the force using the action of probe antibranes 3

2.1 Backreacted D3 branes in the warped deformed conifold 3

2.2 M-theory on a warped Stenzel space 5

$\begin{array}{lll}3 & \text { Computing the force on probe branes } & 6\end{array}$

$\begin{array}{lll}3.1 & \text { Warped deformed conifold } & 6\end{array}$

$\begin{array}{lll}3.2 & \text { M-theory on a warped Stenzel space } & 7\end{array}$

\section{Introduction and motivation}

Anti-D3-branes in warped deformed conifold throats are widely used in string theory model building and string cosmology, both to get de Sitter solutions [1], and to construct string theoretic models of inflation using D3 branes moving in such throats [2].

In a previous work [3], three of us attempted to construct the first-order backreacted supergravity solution for a stack of anti-D3 branes in the Klebanov-Strassler (KS) background [4]. Such antibranes were conjectured in [5] to give rise to holographic duals to metastable vacua of a strongly-coupled gauge theory, and the supergravity analysis implies that the would-be anti-D3 brane solution must have a certain infrared singularity. A similar result was obtained by investigating anti-M2 branes in a warped Stenzel background [6]. If these singularities have a physical origin, then the solutions found in $[3,6]$ describe the first-order backreaction of antibranes in these backgrounds. If these singularities are pathological, the analyses of $[3,6]$ imply that antibranes in backgrounds with positive brane charge dissolved in fluxes cannot be treated in perturbation theory.

In the present work we will work under the assumption that the singularities found in $[3,6]$ are physical, and that antibranes can be treated as perturbations of their respective backgrounds with charge dissolved in fluxes. ${ }^{1}$

In certain string inflation models, the inflaton is the position of a BPS D3 brane in a warped background with anti-D3 branes at its bottom, and the brane-antibrane force gives the derivative of the inflaton potential. There exist two methods to compute this potential. The first, introduced in [2] and widely used in string cosmology constructions, treats the anti-D3 branes as probes in the (easy to find) backreacted solution sourced by BPS D3 branes up the throat. This method involves calculating the change in the potential of the anti-D3 branes as the position of the D3 branes is altered. This yields the force felt by these D3 branes in the warped deformed conifold with anti-D3 branes.

\footnotetext{
${ }^{1}$ Note that this does not automatically imply that antibranes give rise to metastable vacua - for this one would have to show also that the antibrane solution does not contain other non-normalizable modes.
} 
The second method to derive the inflaton potential consists in constructing the firstorder backreacted solution sourced by anti-D3 branes placed at the bottom of a warped deformed conifold [3] and to compute the force felt by a probe D3 brane in this background. Despite the rather complicated nature of the first-order deformation space, the force on a probe D3 turns out to depend only on one of the fourteen integration constants that parametrize the space of $\mathrm{SU}(2) \times \mathrm{SU}(2) \times \mathbb{Z}_{2}$-invariant deformations [3]. Furthermore, the leading large-distance behavior of the inflaton potential agrees with the one computed in [2].

One natural question to ask is whether the two calculations for the inflaton potential agree also beyond leading-order. At first glance, one expects that they should indeed agree. However, the answer does not appear to be so simple. If in the vacuum the calculations of the force using the bare action of one brane in the background of the other should indeed agree, there is no reason this should happen in a background where the charge/anticharge symmetry is broken by the D3 charge dissolved in flux. Indeed, because of harmonic superposition, the fields of the D3 brane are not screened [7]. Yet, there is no reason why the anti-D3 would not be screened by the D3 charge dissolved in flux. Hence, one would expect to have a screening cloud around the anti-D3 branes, which would affect the potential felt by a bare D3 brane. Note that this is a generic phenomenon in media where positive and negative charges are screened differently: because of the different profiles of the screening clouds, the force computed using the action of a bare negative charge in the background of the screened positive charge needs not agree with the force computed using the action of a bare positive charge around the screened negative charge. In the language of plasma physics, the Debye screening lengths of the positive and of the negative charges need not be equal.

The purpose of this letter is to show that the forces computed in the two approaches outlined above agree not only in leading behavior, but in full functional form, modulo a to-be-determined overall normalization constant. This indicates that this force is not screened by the brane charge dissolved in flux. ${ }^{2}$ There are two obvious explanations for this: either anti-D3 branes are not screened by the positive D3 brane charge dissolved in flux, or they are screened, but the screening cloud does not interact with D3 branes. This latter possibility would imply that antibranes change the profile of the cloud of charge dissolved in fluxes, but do not alter its properties, in particular the fact that the local D3 charge density remains equal to the mass density; such a cloud would not interact with probe D3 branes and would not screen the force.

We find no brane-antibrane force screening, both for anti D3-branes at the bottom of the Klebanov-Strassler solution, and for anti-M2 brane at the bottom of a warped Stenzel space with M2 brane charge dissolved in flux [8,9], and hence we believe this is likely a generic phenomenon in flux compactifications. ${ }^{3}$

\footnotetext{
${ }^{2}$ Our analysis does not formally exclude screening by a delta-function-shaped screening cloud, which would keep the same functional expression of the force while changing the overall normalization constant. However, it is hard to believe this is anything but a formal possibility. We leave the actual computation of this constant to a forthcoming publication [10].

${ }^{3}$ In an upcoming paper [11] we will also show this for anti-D2 branes in backgrounds with D2 brane charge dissolved in fluxes [12].
} 
Hence, in an optimistic scenario (if the IR singularities found in [3] and [6] are physical, and we can trust perturbation theory), modulo this subtle issue about the overall constant, our calculation yields the exact functional form of the inflaton potential in a brane/antibrane realization of inflation in string theory. It also demonstrates that the force between branes and antibranes is not screened, and therefore the probe antibrane calculation à la KKLMMT [2] of this inflaton potential in other string inflationary models gives the exact functional form of the potential, not only its leading behavior. This should allow in turn to accurately compute the power spectrum in those models and to compare them with observation.

The paper is organized as follows. In section 2 we review the calculation of the brane/antibrane force, treating the smeared antibranes as probes, both for anti-D3 branes in KS and for anti-M2 branes in a warped Stenzel background $[8,9]$. In section 3 we use the first-order backreacted solutions of [3] and [6] to compute this force using the action of probe D3 and M2 branes, respectively. As advertised, the two calculations agree.

Note. Three months after this paper appeared on the main electronic archive, the preprint arXiv:1102.1734 appeared, which shows that our results can also be obtained out of perturbing a solution with positive charge dissolved in fluxes by a certain function that controls the force on a probe brane (called $\Phi_{-}$), in a manner first discussed in arXiv:0808.2811. Seven months later, the preprint arXiv:1106.6165 appeared, which, in particular, also reproduces the result from the present note by fully solving the equations that give the perturbative anti-D3 brane solution numerically. The advantage of the approach of arXiv:1102.1734 and of the modus operandi of arXiv:1106.6165 is that they yield the exact normalization of the force as well. Namely, they show that not only the functional form of the force but also its overall coefficient is the same as the one stemming from the KKLMMT [2] analysis.

\section{Computing the force using the action of probe antibranes}

To establish whether antibranes are screened by charge dissolved in flux in the warped deformed conifold or the Stenzel space, we first smear them at the tip of those two geometries. This way we preserve the symmetries of the solution without antibranes, and render the calculation of the backreaction of the antibranes an achievable task. The force between the smeared antibranes and the BPS branes at some distance $r=r_{0}$ up the throat will then be the same, by symmetry, as the force between the smeared antibranes and a uniform shell of BPS branes at the same distance.

We demonstrate how to compute the force generated between a stack of antibranes at the bottom of a warped throat and a stack of branes some distance up the throat. This is computed in two ways: either by backreacting the branes while leaving the antibranes as probes; or from backreacting the antibranes and leaving the branes as probes.

\subsection{Backreacted D3 branes in the warped deformed conifold}

To obtain a fully backreacted solution with BPS D3 branes in the warped deformed conifold one simply needs to add to the warp factor a harmonic function (given by the Green's 
function on this Calabi-Yau manifold) sourced by these branes [7]. While in general this is a non-trivial task $[13,14]$, here we are considering smeared branes and as such the Green's function is radially symmetric and the problem is tractable.

The two radially symmetric solutions to the Laplace equation on the deformed conifold are

$$
\begin{aligned}
& H_{1}(\tau)=c_{1} \\
& H_{2}(\tau)=c_{2} \int_{\tau}^{\infty} \frac{d \tau^{\prime}}{\left(\sinh 2 \tau^{\prime}-2 \tau^{\prime}\right)^{2 / 3}}
\end{aligned}
$$

With a shell of D3 branes at $\tau=\tau_{0}$, the full warp factor is

$$
H(\tau)=H_{0}(\tau)+\delta H(\tau)
$$

Here $H_{0}(\tau)$ is the zeroth-order warp factor for the warped deformed conifold:

$$
\begin{aligned}
H_{0} & =e^{-4 A_{0}-4 p_{0}+2 x_{0}} \\
& =h_{0}-32 P^{2} \int_{0}^{\tau} \frac{t \operatorname{coth} t-1}{\sinh ^{2} t}\left(\frac{1}{2} \sinh (2 t)-t\right)^{1 / 3} d t,
\end{aligned}
$$

where $P$ is the RR three-form flux through the $S^{3}$ of the deformed conifold, and $h_{0}$ is a constant. ${ }^{4}$ On top of the warp factor for the zeroth-order solution, there is the following contribution from the $N$ D3 branes at $\tau=\tau_{0}$ :

$$
\delta H(\tau)=\left\{\begin{array}{l}
H_{1}(\tau), \tau<\tau_{0}, \\
H_{2}(\tau), \tau>\tau_{0} .
\end{array}\right.
$$

The two integration constants $\left(c_{1}, c_{2}\right)$ are related by matching at the source the solutions in the two domains above:

$$
c_{1}=H_{2}\left(\tau_{0}\right) .
$$

To fix the other integration constant in terms of the number of D3 branes, we rely on the standard quantization formula for the five-form field strength:

$$
\frac{1}{\left(4 \pi^{2} \alpha^{\prime}\right)^{2}} \int F_{5}=N
$$

and integrate on the $T^{1,1}$ surfaces right outside and right inside the shell using

$$
g_{s} F_{5}=*_{10} d H^{-1} \wedge d x_{0} \wedge \ldots \wedge d x_{3} .
$$

The difference of the two integrals gives the D3 brane charge of the shell and its relation to the coefficient in $\delta H$ :

$$
c_{2}=4 \pi\left(\frac{2^{1 / 3} \alpha^{\prime}}{\varepsilon^{4 / 3}}\right)^{2} g_{s} N
$$

where we use the conventions of [15].

\footnotetext{
${ }^{4}$ Explicitly, we have $h_{0}=32 P^{2} \int_{0}^{\infty} \frac{\tau \operatorname{coth} \tau-1}{\sinh ^{2} \tau}\left(\frac{1}{2} \sinh (2 \tau)-\tau\right)^{1 / 3} d \tau=18.2373 P^{2}$.
} 
We now compute the potential of probe anti-D3 branes placed at the tip of the cone. Since for a BPS D3 brane the DBI and WZ potentials cancel, for anti-D3 branes these potentials are equal in magnitude and sign:

$$
V_{\overline{\mathrm{D} 3}}=V_{\mathrm{DBI}}+V_{\mathrm{WZ}}=2 V_{\mathrm{WZ}}
$$

Expanding the potential to first-order in the number of D3 branes we find

$$
\begin{aligned}
V_{\overline{\mathrm{D} 3}} & =2 H^{-1} \\
& =2 H_{0}^{-1}\left(1-\frac{\delta H}{H_{0}}\right)+\mathcal{O}\left((N / P)^{2}\right) .
\end{aligned}
$$

The force exerted by the anti-D3 branes on the D3 branes can then be obtained from the variation of this potential as the source D3 branes are moved [2]

$$
\begin{aligned}
F_{\mathrm{D} 3} & =-\left.\frac{\partial V_{\overline{\mathrm{D}}}}{\partial \tau_{0}}\right|_{\tau=0} \\
& =-\frac{1}{\left.H_{0}^{2}\right|_{\tau=0}} \frac{c_{2}}{\left(\sinh 2 \tau_{0}-2 \tau_{0}\right)^{2 / 3}} .
\end{aligned}
$$

The dependence of this force on $N$ appears through the constant $c_{2}(2.9)$.

\subsection{M-theory on a warped Stenzel space}

The generalization of the probe brane computation of Kachru, Pearson and Verlinde [5] to a warped Stenzel space M-theory background [8, 9] has recently been performed in [16]. Motivated by this analysis, three of the authors have used the technology of [3] to study the backreaction of anti-M2 branes in this space [6]. The probe brane analysis of the previous section can also be performed, and we find that although the Green's function itself is a complicated combination of incomplete elliptic integrals:

$$
\begin{aligned}
H_{1}(y)= & d_{1}, \\
H_{2}(y)= & \frac{2}{45} d_{2}\left[\frac{9 \sqrt{y^{4}-1}}{y^{5}}+3 E(\arcsin (1 / y) \mid-1)-3 F(\arcsin (1 / y) \mid-1)\right. \\
& +5 \sqrt{3}(\Pi(\sqrt{3} ;-\arcsin (1 / y) \mid-1)-\Pi(-\sqrt{3} ;-\arcsin (1 / y) \mid-1))],
\end{aligned}
$$

with $d_{i}$ integration constants and $k$ a constant that ensures that $H_{2}$ vanishes at large $y$, the derivative of this Green's function is very simple: ${ }^{5}$

$$
H_{2}^{\prime}(r)=\frac{3 \sqrt{2} d_{2} \operatorname{csch}^{3} r}{(2+\cosh 2 r)^{3 / 4}}
$$

From flux quantization

$$
\frac{1}{\left(2 \pi \ell_{p}\right)^{6}} \int_{V_{5,2}} *_{11} G_{4}=N
$$

\footnotetext{
${ }^{5}$ The standard coordinate we use is $y^{4}=2+\cosh 2 r$.
} 
with

$$
G_{4}=d H^{-1} \wedge d x_{0} \wedge d x_{1} \wedge d x_{2},
$$

we find that the M2 brane charge of the shell, $N$, is related to the constant in the new warp factor via

$$
d_{2}=(2 \pi)^{2} \ell_{p}^{6} \sqrt{2} N .
$$

In addition, there is the matching condition

$$
d_{1}=H_{2}\left(y_{0}\right) .
$$

If we now consider the change in the potential of probe antibranes with the position of the source M2 branes in this background, we obtain the force:

$$
F_{M 2}=-\frac{1}{\left.H_{0}^{2}\right|_{r=0}} \frac{3 \sqrt{2} d_{2} \operatorname{csch}^{3} r_{0}}{\left(2+\cosh 2 r_{0}\right)^{3 / 4}} .
$$

\section{Computing the force on probe branes}

\subsection{Warped deformed conifold}

We now use the results from [3] and refer to this work for much of the notation. In that paper three of the authors found that the force felt by a probe D3 brane in the first-order deformed KS background has the remarkably-simple form

$$
F_{\mathrm{D} 3}=\frac{2}{3} e^{-2 x_{0}} \tilde{\xi}_{1},
$$

where $\tilde{\xi}_{1}$ is one of the sixteen modes parameterizing the deformation space ${ }^{6}[22]$ and is given by

$$
\tilde{\xi}_{1}=\widetilde{X}_{1} \exp \left(\int_{0}^{\tau} d \tau^{\prime} e^{-2 x_{0}}\left[2 P f_{0}-F_{0}\left(f_{0}-k_{0}\right)\right]\right) .
$$

Here $X_{1}$ is an integration constant and

$$
\begin{aligned}
e^{x_{0}} & =\frac{1}{4} H_{0}^{1 / 2}\left(\frac{1}{2} \sinh (2 \tau)-\tau\right)^{1 / 3}, \\
f_{0} & =-P \frac{(\tau \operatorname{coth} \tau-1)(\cosh \tau-1)}{\sinh \tau}, \\
k_{0} & =-P \frac{(\tau \operatorname{coth} \tau-1)(\cosh \tau+1)}{\sinh \tau}, \\
F_{0} & =P \frac{(\sinh \tau-\tau)}{\sinh \tau},
\end{aligned}
$$

with $H_{0}$ given in (2.4).

We make great use of the simple yet elusive observation that this integral can in fact be performed exactly

$$
\begin{aligned}
\tilde{\xi}_{1} & =\widetilde{X}_{1} \exp \left(\int_{0}^{\tau} d \tau^{\prime} \frac{H_{0}^{\prime}}{H_{0}}\right) \\
& =X_{1} H_{0}(\tau) .
\end{aligned}
$$

\footnotetext{
${ }^{6}$ This deformation space has been considered previously in various respects [17-21].
} 
The force now takes the form

$$
\begin{aligned}
F_{\mathrm{D} 3} & =\frac{2}{3} e^{-2 x_{0}} X_{1} H_{0}(\tau) \\
& =\frac{32}{3} \frac{2^{2 / 3} X_{1}}{(\sinh 2 \tau-2 \tau)^{2 / 3}} .
\end{aligned}
$$

Remarkably enough, this has exactly the same functional form as the force computed in (2.12) using the probe antibrane potential. As mentioned in the Introduction, the fact that the two calculations of the force agree implies that this force is not screened by the positive D3 brane charge dissolved in flux.

As has been explained in [3] the value of $X_{1}$ can be determined in terms of the UV and IR boundary conditions, but this requires relating the UV and IR values of all sixteen integration constants involved in the full solution, which can only be done numerically. Once this numerical work is completed, we will be able to compare the coefficient of the force computed in this section with the calculation of section 2.1. Whether these two numbers agree or not will help elucidate the physics of anti-D3 branes in the KlebanovStrassler background. We plan to report on these results soon [10].

\subsection{M-theory on a warped Stenzel space}

The same steps for M-theory on a Stenzel space have recently been performed in [6] and we merely quote the results and refer to this work for the notation. When considering the candidate backreacted solution corresponding to anti-M2 branes, the force felt by a probe M2 brane is

$$
\begin{aligned}
F & =-\frac{2}{3} e^{-3\left(\alpha_{0}+\beta_{0}\right)(r)} e^{-3 z_{0}(0)} X_{4} \\
& =-\frac{18 e^{-3 z_{0}(0)} X_{4} \operatorname{csch}^{3} r}{(2+\cosh 2 r)^{3 / 4}} .
\end{aligned}
$$

This has again the same functional form as (2.18), up to the determination of the integration constant $X_{4}$ in terms of the charges of the system. This demonstrates that, much like in the anti-D3 brane story, the force between anti-M2 branes and M2 branes is not screened by the charge dissolved in flux.

\section{Acknowledgments}

We would like thank Jean-Paul Blaizot and Jean-Yves Ollitrault for informative discussions. The work of G. G. is supported by a Contrat de Formation par la Recherche of CEA/Saclay. The work of I. B., M. G. and N. H. is supported by the DSM CEA/Saclay, the grant ANR07-CEXC-006, the ANR grant 08-JCJC-0001-0, and by the ERC Starting Independent Researcher Grant 240210 - String-QCD-BH.

Open Access. This article is distributed under the terms of the Creative Commons Attribution License which permits any use, distribution and reproduction in any medium, provided the original author(s) and source are credited. 


\section{References}

[1] S. Kachru, R. Kallosh, A.D. Linde and S.P. Trivedi, de Sitter vacua in string theory, Phys. Rev. D 68 (2003) 046005 [hep-th/0301240] [INSPIRE].

[2] S. Kachru et al., Towards inflation in string theory, JCAP 10 (2003) 013 [hep-th/0308055] [INSPIRE].

[3] I. Bena, M. Graña and N. Halmagyi, On the existence of meta-stable vacua in Klebanov-Strassler, JHEP 09 (2010) 087 [arXiv:0912.3519] [INSPIRE].

[4] I.R. Klebanov and M.J. Strassler, Supergravity and a confining gauge theory: duality cascades and chi SB resolution of naked singularities, JHEP 08 (2000) 052 [hep-th/0007191] [INSPIRE].

[5] S. Kachru, J. Pearson and H.L. Verlinde, Brane/flux annihilation and the string dual of a nonsupersymmetric field theory, JHEP 06 (2002) 021 [hep-th/0112197] [INSPIRE].

[6] I. Bena, G. Giecold and N. Halmagyi, The backreaction of anti-M2 branes on a warped Stenzel space, JHEP 04 (2011) 120 [arXiv: 1011.2195] [INSPIRE].

[7] M. Graña and J. Polchinski, Gauge/gravity duals with holomorphic dilaton, Phys. Rev. D 65 (2002) 126005 [hep-th/0106014] [INSPIRE].

[8] M. Stenzel, Ricci-flat metrics on the complexification of a compact rank one symmetric space, Manuscripta Math. 80 (1993) 151.

[9] M. Cvetič, G. Gibbons, H. Lü and C. Pope, Ricci flat metrics, harmonic forms and brane resolutions, Commun. Math. Phys. 232 (2003) 457 [hep-th/0012011] [INSPIRE].

[10] I. Bena, G. Giecold, M. Grana and N. Halmagyi, work in progress.

[11] G. Giecold et al., to appear.

[12] M. Cvetič, G. Gibbons, H. Lü and C. Pope, Supersymmetric nonsingular fractional D-2 branes and NS-NS 2 branes, Nucl. Phys. B 606 (2001) 18 [hep-th/0101096] [INSPIRE].

[13] C. Krishnan and S. Kuperstein, The mesonic branch of the deformed conifold, JHEP 05 (2008) 072 [arXiv:0802.3674] [INSPIRE].

[14] S.S. Pufu, I.R. Klebanov, T. Klose and J. Lin, Green's Functions and Non-Singlet Glueballs on Deformed Conifolds, J. Phys. A 44 (2011) 055404 [arXiv:1009.2763] [InSPIRE].

[15] C.P. Herzog, I.R. Klebanov and P. Ouyang, D-branes on the conifold and $N=1$ gauge/gravity dualities, hep-th/0205100 [INSPIRE].

[16] I.R. Klebanov and S.S. Pufu, M-Branes and metastable states, JHEP 08 (2011) 035 [arXiv: 1006.3587] [INSPIRE].

[17] S. Kuperstein and J. Sonnenschein, Analytic nonsupersymmtric background dual of a confining gauge theory and the corresponding plane wave theory of hadrons, JHEP 02 (2004) 015 [hep-th/0309011] [INSPIRE].

[18] M. Berg, M. Haack and W. Mueck, Bulk dynamics in confining gauge theories, Nucl. Phys. B 736 (2006) 82 [hep-th/0507285] [INSPIRE].

[19] M. Berg, M. Haack and W. Mueck, Glueballs vs. Gluinoballs: Fluctuation Spectra in Non-AdS/Non-CFT, Nucl. Phys. B 789 (2008) 1 [hep-th/0612224] [INSPIRE].

[20] M.K. Benna, A. Dymarsky, I.R. Klebanov and A. Solovyov, On normal modes of a warped throat, JHEP 06 (2008) 070 [arXiv:0712.4404] [INSPIRE]. 
[21] A. Dymarsky, D. Melnikov and A. Solovyov, I-odd sector of the Klebanov-Strassler theory, JHEP 05 (2009) 105 [arXiv:0810.5666] [INSPIRE].

[22] V. Borokhov and S.S. Gubser, Non-supersymmetric deformations of the dual of a confining gauge theory, JHEP 05 (2003) 034 [hep-th/0206098] [INSPIRE]. 